\title{
COMPOSIÇÃO E CARACTERIZAÇÃO DA FAUNA DE MAMÍFEROS DE MÉDIO E GRANDE PORTE EM UMA PEQUENA RESERVA DE CERRADO EM MATO GROSSO, BRASIL ${ }^{1}$
}

\author{
Ednaldo Cândido Rocha² e Julio César Dalponte ${ }^{3}$
}

\begin{abstract}
RESUMO - Este estudo foi realizado na Reserva Biológica Municipal "Mário Viana”, Nova Xavantina, MT, objetivando inventariar e avaliar a abundância e diversidade de mamíferos terrestres de médio e grande porte. Para tanto, foram realizadas duas visitas mensais a um transecto com $2.820 \mathrm{~m}$ de extensão, durante todo o ano de 2001, para o levantamento de pegadas (rastreamentos) e outras evidências de mamíferos. Um total de 29 espécies foram registradas na área de estudo, sendo que 22 ocorreram no transecto e tiveram suas sequiências individuais de pegadas quantificadas para realização do cálculo dos índices de abundância e de diversidade de Shannon-Wiener $\left(H^{\prime}\right)$. De acordo com seus índices de abundância, as espécies foram classificadas em raras, comuns e abundantes. Dentre outras, onça-parda (Puma concolor - Linnaeus, 1771) e tatu-canastra (Priodontes maximus - Keer, 1792) mostraram-se raras; jaguatirica (Leopardus pardalis - Linnaeus, 1758), e tamanduábandeira (Myrmecophaga tridactyla - Linnaeus, 1758), comuns; e cutia (Dasyprocta azarae - Lichtenstein, 1823) e tapeti (Sylvilagus brasiliensis - Linnaeus, 1758), abundantes. O $H^{\prime}$ 'encontrado foi 2,40 , sendo considerado significativo. O presente trabalho apontou que, apesar de pequena (470 ha), a área de estudo desempenha importante papel para a conservação da mastofauna da região de Nova Xavantina, MT.
\end{abstract}

Palavras-chave: Conservação, mastofauna e rastreamento.

\section{COMPOSITION AND CHARACTERIZATION OF THE MEDIUM AND LARGE SIZE MAMMAL FAUNA IN A SMALL CERRADO RESERVE IN MATO GROSSO, BRAZIL}

\begin{abstract}
This study was carried out at the 'Mario Viana' Municipal Biological Reserve in Nova Xavantina, MT, aiming to make an inventory and evaluate the abundance and diversity of terrestrial mammals of medium and large size. Thus, two monthly visits were made to a $2.820 \mathrm{~m}$ long transect throughout 2001 to assess tracking and other evidences of mammals. Twenty-nine species were recorded in the study area, with 22 in the transect and two individual tracking sequences being quantified for calculation of the Shannon-Wiener ( $\left.\mathrm{H}^{\prime}\right)$ abundance and diversity indices. According to the abundance indices, the species were classified as rare, common and abundant. Among other species, onça-parda (Puma concolor (Linnaeus, 1771)) and tatucanastra (Priodontes maximus (Keer, 1792) were classified as rare; jaguatirica (Leopardus pardalis (Linnaeus, 1758)) and tamanduá-bandeira (Myrmecophaga tridactyla Linnaeus, 1758) as common; and cutia (Dasyprocta azarae (Lichtenstein, 1823)) and tapetí (Sylvilagus brasiliensis (Linnaeus, 1758)) as abundant species. The $\mathrm{H}^{\prime}$ index found was 2.40, which was considered significant. This work shows that despite being small (470 ha), the area studied plays an important role in the preservation of the mammalian fauna in the region of Nova Xavantina, MT.
\end{abstract}

Keywords: mammalian fauna, preservation and tracking.

\footnotetext{
${ }^{1}$ Recebido em $1^{\circ} .04 .2005$ e aceito para publicação em 05.04.2006.

${ }^{2}$ Programa de Pós-Graduação em Ciência Florestal, Universidade Federal de Viçosa. Endereço: Rua São Paulo, n ${ }^{\circ} 375$, Centro, Campinápolis - MT, CEP 78630-000. E-mail: <ednaldorocha@yahoo.com.br>.

${ }^{3}$ Departamento de Ciências Biológicas, Universidade do Estado de Mato Grosso, Campus de Nova Xavantina - MT.
} 


\section{INTRODUÇÃO}

Dos mamíferos descritos atualmente, cerca de 524 espécies ocorrem em território brasileiro, o que representa cerca de $13 \%$ da mastofauna do mundo. Esses números fazem com que o Brasil possua a maior riqueza de mamíferos de toda a região neotropical (FONSECA et al., 1996). Os mamíferos com ocorrência no Cerrado totalizam cerca de 195 espécies, sendo que 18 delas são endêmicas e 17 estão incluídas na lista nacional das espécies da fauna brasileira ameaçadas de extinção (MMA, 2003).

O grau de ameaça e a importância ecológica do grupo tornam evidente a necessidade de incluir informações sobre os mamíferos terrestres de médio e grande porte em inventários e diagnósticos ambientais (PARDINI et al., 2003).

Dados sobre abundância e distribuição são muito importantes para a avaliação do status de conservação de um táxon (IUCN, 2001), sendo que uma das dificuldades para essa avaliação em nível nacional é a escassez de dados publicados sobre composição e abundância das espécies em níveis locais e regionais. Apenas os Estados do Paraná, de Minas Gerais, São Paulo, do Rio de Janeiro e Rio Grande do Sul possuem listas de espécies de mamíferos ameaçados de extinção.

Os primeiros registros sobre a mastofauna da região de Nova Xavantina, leste do Estado de Mato Grosso, foram realizados durante a expedição anglo-brasileira Xavantina-Cachimbo no final da década de 1960 (PINE et al., 1970). Posteriormente, outros trabalhos sobre mamíferos também foram desenvolvidos nessa região (WWF, 2000; DALPONTE, 2003). No entanto, a única Unidade de Conservação presente no Município de Nova Xavantina (Reserva Biológica Municipal "Mário Viana" - RBMMV), com cerca de 470 ha, ainda não dispõe de trabalhos publicados sobre sua mastofauna.

Este trabalho objetivou inventariar e avaliar a abundância e diversidade de mamíferos terrestres, de médio e grande porte, na RBMMV, destacando-se informações relevantes sobre a situação local das espécies.

\section{MATERIAL E MÉTODOS}

\section{1. Área de estudo}

O estudo foi realizado na Reserva Biológica Municipal "Mário Viana" (RBMMV), 1442' S e 52²1'
W, criada pela Lei Municipal no 652, de 27/12/1995. Trata-se de um fragmento de Cerrado que possui uma área de aproximadamente $470 \mathrm{ha}$, distante $5 \mathrm{~km}$ do centro da cidade de Nova Xavantina, onde está localizado o Campus da Universidade do Estado de Mato Grosso - UNEMAT.

O clima da região de Nova Xavantina é do tipo Aw, segundo a classificação de Köppen, caracterizado por duas estações bem definidas, uma seca (de abril a setembro) e uma chuvosa (de outubro a março), sendo a precipitação pluviométrica média anual de $1.600 \mathrm{~mm}$ (NIMER, 1989). O relevo é caracterizado como plano a ondulado, altitudes entre 250 e $300 \mathrm{~m}$ e com ocorrências de solos litólicos, cambissolo transicional com deposição de blocos de arenito e Latossolo Vermelho-Amarelo (RADAMBRASIL, 1981).

A RBMMV possui fisionomias vegetais características do bioma Cerrado, com o predomínio do cerrado sensu stricto. Ocorrem também formações como cerradão, campo limpo e mata de galeria (FELFILI et al., 1998; MARIMON et al., 1998). Impactos anteriores, resultantes da extração de madeira e implantação de pastagem exótica não ocorrem mais, o que tem permitido um processo de recuperação da área após a implantação do Campus da UNEMAT. Atualmente, a área tem sido alvo de vários projetos visando à conservação dos meios físico e biótico.

\subsection{Coleta dos dados}

Os dados foram coletados no período de janeiro a dezembro de 2001, realizando-se duas visitas mensais a um transecto com $2.820 \mathrm{~m}$ de extensão, o que totalizou $67.680 \mathrm{~m}$ percorridos, durante 24 rastreamentos (identificação e interpretação de pegadas). Além do transecto, toda a área da RBMMV foi aleatoriamente percorrida em busca de evidências de mamíferos.

A amostragem de mamíferos incluiu métodos indireto e direto para o registro das espécies, conforme apresentado a seguir:

1. Métodos indiretos: mamíferos de médio e grande porte foram identificados e tentativamente quantificados pelo levantamento de pegadas (rastreamentos). A cada visita, o transecto foi rigorosamente inspecionado e, quando encontradas pegadas, estas foram identificadas (BECKER e DALPONTE, 1991) e as informações organizadas para constituir a lista de espécies da área amostrada, bem como sua abundância relativa e índice 
de diversidade. Adicionalmente, sinais acústicos, fezes e tocas foram utilizados para a detecção de mamíferos nos sítios amostrados.

2. Método direto: mamíferos de médio e grande porte também foram detectados por visualização durante percursos a pé ao longo da RBMMV.

Os rastreamentos foram feitos sempre pela manhã (a partir de $7 \mathrm{~h}$ ) e demoravam cerca de $1 \mathrm{~h} 30$ cada. Para evitar recontagem de rastros dos dias anteriores, procedeu-se da seguinte forma: a) na estação chuvosa, os rastreamentos aconteceram na primeira manhã após uma tarde de chuva (BROWER e ZAR, 1984); e, na estação seca, as pegadas velhas existentes no transecto foram apagadas, arrastando-se vassouras e palhas, na tarde anterior ao dia de coleta de dados.

A contagem de seqüências de pegadas foi procedida cuidando-se para que pegadas de um animal não fossem recontadas durante a amostragem, sendo utilizados os seguintes critérios para a individualização destes: a direção tomada pelo animal, o tamanho das pegadas e a distância entre as seqüências de pegadas.

Na tentativa de compor uma lista completa das espécies que ocorrem na RBMMV, foram considerados também os registros do lobo-guará (Chrysocyon brachyurus (Illiger, 1815)), em 2004 (GODOY, comunicado pessoal), e do quati (Nasua nasua (Linnaeus, 1766)) e ouriço (Couendou prehensilis (Linnaeus, 1758)), ambas em 2000 (DALPONTE, observação pessoal).

De acordo com a definição de Hayward e Phillipson (1979), mamíferos de pequeno porte são aqueles que não ultrapassam o peso de $5 \mathrm{~kg}$ quando adultos. Dessa forma, algumas espécies listadas no presente estudo não são de médio e grande porte, como gambá (Didelphis albiventris Lund, 1840), tatu-de-rabo-mole (Cabassous unicinctus (Linnaeus, 1758)), tatu-galinha (Dasypus novemcinctus Linnaeus, 1758), macaco-prego (Cebus libidinosus Spix, 1823), raposa-do-campo (Pseudalopex vetulus (Lund, 1842)), irara (Eira barbara (Linnaeus, 1758)), gato-mourisco (Herpailurus yaguarondi (Lacépède, 1809)), gato-do-mato-pequeno (Leopardus tigrinus (Schreber, 1775)), ouriço (Coendou prehensilis (Linnaeus, 1758)), preá (Cavia aperea Erxleben, 1777), cutia (Dasyprocta azarae Lichtenstein, 1823) e tapeti (Sylvilagus brasiliensis (Linnaeus, 1758)), mas foram incluídas neste trabalho, pois puderam ser seguramente identificadas na área de estudo. O peso médio dos animais foi consultado em Fonseca et al. (1996).

\subsection{Análise dos dados}

A identificação e contagem de pegadas têm sido usada como índice de abundância, sobretudo para as espécies que são de difícil visualização ou captura (WILKIE e FINN, 1990; NICHOLS e CONROY, 1996; CUELLAR e NOSS, 1997; STANDER etal., 1997; DOTTA, 2005). Para tanto, alguns pesquisadores utilizam o registro de pegadas em parcelas (plots) de areia previamente preparadas e, muitas vezes, usando iscas para atrair os animais (PARDINI et al., 2003; SCOSS et al., 2004). No entanto, neste estudo buscou-se uma forma de apresentar a abundância relativa das espécies criando um índice individual de pegadas por $\mathrm{km}$ percorrido, já que a área dispunha de um transecto (estrada) contínuo, passível de rastreamento em grande parte de sua extensão.

Para o cálculo do índice de abundância relativa, o número de seqüências individuais de pegadas de cada espécies foi dividido pelo total de quilômetros percorridos, em cada rastreamento. Assim, obteve-se o número médio de seqüências individuais de pegadas por $\mathrm{km}$ percorrido.

Uma vez calculada a abundância relativa, procedeuse à avaliação do status local das espécies de mamíferos. Para tanto, foram estabelecidas, com base em experiência adquirida em trabalhos práticos na própria região, três classes de distribuição das espécies, conforme os seguintes critérios: (1) espécies raras - as que apresentaram até 0,10 seqüência individual de pegadas por km percorrido; (2) espécies comuns - as que apresentaram de 0,11 a 0,50 seqüência individual de pegadas por km percorrido; e (3) espécies abundantes - as que apresentaram acima de 0,50 seqüência individual de pegadas por $\mathrm{km}$ percorrido.

A partir dos dados de abundância amostrados no transecto, foi calculado o Índice de diversidade de Shannon-Wienner ( $H^{\prime}$ ) (LUDWIG e REYNOLDS, 1988; MAGURRAN, 1988):

$$
H^{\prime}=-\sum_{i=1}^{s}\left(\frac{n_{i}}{N} \cdot \ln \frac{n_{i}}{N}\right)
$$

em que, $H^{\prime}$ é o índice de diversidade, $n i$ é o número de registros da espécie $i$ e $N$ o número total de registros.

R. Árvore, Viçosa-MG, v.30, n.4, p.669-678, 2006 


\section{RESULTADOS E DISCUSSÃO}

\subsection{Riqueza de espécies}

Foram obtidos registros de 29 espécies de mamíferos (um marsupial, seis xenartros, dois primatas, 10 carnívoros, um perissodáctilo, três artiodáctilos, cinco roedores e um lagomorfo), sendo que seis dessas estão ameaçadas de extinção (MMA, 2003). A lista apresentada neste estudo foi composta por espécies detectadas, principalmente por pegadas diagnósticas e secundariamente por outras observações indiretas (como vocalizações, tocas e fezes) e diretas (visualizações) (Quadro 1).

Quadro 1 - Taxa (ordem, família e espécie), nome comum e tipo de registros de mamíferos encontrados na Reserva Biológica Municipal "Mário Viana”, Nova Xavantina, MT

Table 1 -Taxa (order, family and species), common name and type records of mammals found in the 'Mario Viana' Municipal Biological Reserve, Nova Xavantina, MT

\begin{tabular}{|c|c|c|}
\hline Taxa & Nome comum & Registro \\
\hline \multicolumn{3}{|l|}{ Didelphimorphia } \\
\hline \multicolumn{3}{|l|}{ Didelphidae } \\
\hline Didelphis albiventris Lund, 1840 & gambá, mucura & visualização e pegadas \\
\hline \multicolumn{3}{|l|}{ Xenarthra } \\
\hline \multicolumn{3}{|l|}{ Myrmecophagidae } \\
\hline Myrmecophaga tridactyla Linnaeus, 1758* & tamanduá-bandeira & pegadas e fezes \\
\hline Tamandua tetradactyla (Linnaeus, 1758) & tamanduá-mirim & pegadas \\
\hline \multicolumn{3}{|l|}{ Dasypodidae } \\
\hline Cabassous unicinctus (Linnaeus, 1758) & tatu-de-rabo-mole & visualização e tocas \\
\hline Dasypus novemcinctus Linnaeus, 1758 & tatu-galinha & visualização, pegadas e tocas \\
\hline Euphractus sexcinctus (Linnaeus, 1758) & tatu-peba, tatu-peludo & visualização, pegadas e tocas \\
\hline Priodontes maximus (Kerr, 1792)* & tatu-canastra & pegadas \\
\hline \multicolumn{3}{|l|}{ Primates } \\
\hline \multicolumn{3}{|l|}{ Cebidae } \\
\hline Alouatta caraya (Humboldt, 1812) & bugio, guariba & visualização e vocalização \\
\hline Cebus libidinosus Spix, 1823 & macaco-prego & visualização \\
\hline \multicolumn{3}{|l|}{ Carnivora } \\
\hline \multicolumn{3}{|l|}{ Canidae } \\
\hline Cerdocyon thous (Linnaeus, 1766) & cachorro-do-mato & pegadas \\
\hline Chrysocyon brachyurus (Illiger, 1815)* & lobo-guará & pegadas \\
\hline Pseudalopex vetulus (Lund, 1842) & raposa-do-campo & pegadas \\
\hline \multicolumn{3}{|l|}{ Procyonidae } \\
\hline Nasua nasua (Linnaeus, 1766) & quati & visualização \\
\hline Procyon cancrivorus (G. Cuvier, 1798) & mão-pelada & pegadas \\
\hline \multicolumn{3}{|l|}{ Mustelidae } \\
\hline Eira barbara (Linnaeus, 1758) & irara, papa-mel & pegadas \\
\hline \multicolumn{3}{|l|}{ Felidae } \\
\hline Herpailurus yaguarondi (Lacépède, 1809) & gato-mourisco & pegadas \\
\hline Leopardus pardalis (Linnaeus, 1758)* & jaguatirica & pegadas \\
\hline Leopardus tigrinus (Schreber, 1775)* & gato-do-mato-pequeno & pegadas, crânio e pele \\
\hline Puma concolor (Linnaeus, 1771)* & onça-parda, suçuarana & pegadas \\
\hline \multicolumn{3}{|l|}{ Perissodactyla } \\
\hline \multicolumn{3}{|l|}{ Tapiridae } \\
\hline Tapirus terrestris (Linnaeus, 1758) & anta & pegadas \\
\hline \multicolumn{3}{|l|}{ Artiodactyla } \\
\hline \multicolumn{3}{|l|}{ Tayassuidae } \\
\hline Pecari tajacu (Linnaeus, 1758) & cateto, caititu & pegadas \\
\hline \multicolumn{3}{|l|}{ Cervidae } \\
\hline Mazama americana (Erxleben, 1777) & veado-mateiro & pegadas \\
\hline Mazama gouazoubira (G. Fischer, 1814) & veado-catingueiro & visualização e pegadas \\
\hline
\end{tabular}

R. Árvore, Viçosa-MG, v.30, n.4, p.669-678, 2006 
Quadro 1 - Cont.

Table 1 - Cont.

\begin{tabular}{|c|c|c|}
\hline Taxa & Nome comum & Registro \\
\hline \multicolumn{3}{|l|}{ Rodentia } \\
\hline \multicolumn{3}{|l|}{ Erethizontidae } \\
\hline Coendou prehensilis (Linnaeus, 1758) & ouriço, porco-espinho & espinhos em fezes de jaguatirica \\
\hline \multicolumn{3}{|l|}{ Caviidae $r$} \\
\hline Cavia aperea Erxleben, 1777 & preá & visualização \\
\hline \multicolumn{3}{|l|}{ Hydrochaeridae } \\
\hline Hydrochaeris hydrochaeris (Linnaeus, 1766) & capivara & pegadas \\
\hline \multicolumn{3}{|l|}{ Cuniculidae } \\
\hline Cuniculus paca (Linnaeus, 1766) & paca & pegadas \\
\hline \multicolumn{3}{|l|}{ Dasyproctidae } \\
\hline Dasyprocta azarae Lichtenstein, 1823 & cutia & visualização e pegadas \\
\hline \multicolumn{3}{|l|}{ Lagomorpha } \\
\hline \multicolumn{3}{|l|}{ Leporidae } \\
\hline Sylvilagus brasiliensis (Linnaeus, 1758) & tapetí, coelho & visualização e pegadas \\
\hline
\end{tabular}

* Espécies ameaçadas de extinção (MMA, 2003).

* Threatened of extinction species (MMA, 2003).

Apesar de não ter sido registrada no presente estudo, é esperado que tatu-mulita (Dasypus septemcinctus Linnaeus, 1758) também ocorra, em baixas densidades, na área da RBMMV, pois essa espécie já foi encontrada em áreas periféricas da cidade de Nova Xavantina (ROCHA, observação pessoal).

A RBMMV, apesar de pequena (470 ha), apresenta uma riqueza de espécies relativamente elevada, pois, comparando com um trabalho realizado em áreas de Floresta Atlântica no Espírito Santo, Chiarello (1999) registrou em duas reservas de tamanho médio (1.504 e 2.400 ha) 30 espécies de mamíferos em cada. Resultados obtidos com pequenos mamíferos não-voadores indicam que Cerrado e Floresta Atlântica são similares quanto à riqueza de espécies de fauna (BONVICINO et al., 2002).

Conforme observado por Chiarello (1999), em reservas de Floresta Atlântica o número de espécies de mamíferos cresce consideravelmente com o aumento do tamanho das reservas. No entanto, distúrbios causados por seres humanos são considerados como o principal fator que determina diferenças na riqueza e abundância de mamíferos no leste da Amazônia (LOPES e FERRARI, 2000).

\subsection{Abundância de espécies}

Das 29 espécies registradas na RBMMV, 22 ocorreram no transecto e tiveram suas seqüências individuais de pegadas quantificadas para o cálculo do índice de abundância relativa, dado pela média de seqüências individuais de pegadas por $\mathrm{km}$ percorrido (Quadro 2). De acordo com seus índices de abundância, as espécies de mamíferos da RBMMV foram classificadas em três grupos: espécies raras, comuns e abundantes.

\subsubsection{Espécies raras}

De acordo com os índices de abundância apresentados no Quadro 2, as espécies mais raras na RBMMV foram onça-parda (Puma concolor (Linnaeus, 1771)), capivara (Hydrochaeris hydrochaeris (Linnaeus, 1776)), tatu-canastra (Priodontes maximus (Keer, 1792)) e raposa-do-campo (Pseudalopex vetulus); as duas primeiras com 0,01 e as duas últimas com 0,03 seqüência individual de pegadas por $\mathrm{km}$ percorrido. Onça-parda e capivara possivelmente não vivem na RBMMV e apenas utilizaram a área como parte de suas áreas de vida ou como refúgio durante alguns de seus deslocamentos. Isso se deve ao fato de que a reserva não dispõe de área com tamanho suficiente para manter um predador de topo de cadeia, como a onça-parda, e também não possui "habitat" adequado à ocorrência de capivara (MARINHO-FILHO et al., 1998), já que apresenta apenas um pequeno córrego intermitente. Quanto ao tatucanastra, a abundância encontrada neste estudo (espécie rara) parece refletir seu padrão de distribuição nacional, considerando-se que esta espécie já foi extinta em muitas áreas onde ocorria, pois é um animal muito visado por caçadores e sensível a fortes distúrbios, como desmatamento e agricultura (MARINHO-FILHO et al., 1998). No entanto,

R. Árvore, Viçosa-MG, v.30, n.4, p.669-678, 2006 
indícios desta espécie são comumente encontrados no Parque Nacional das Emas (RODRIGUES et al., 2002). Para raposa-do-campo, listada como localmente rara, o índice de abundância está subestimado, pois esta espécie vive preferencialmente em "habitats" abertos, como pastagens (DALPONTE, 1997; 2003), que não são comuns na área amostrada.

Tamanduá-mirim (Tamandua tetradactyla (Linnaeus, 1758)), veado-mateiro (Mazama americana (Erxleben, 1777)), gato-mourisco (Herpailurus yaguarondi), mãopelada (Procyon cancrivorus (G. Cuvier, 1798)) e irara (Eira barbara) também se mostraram localmente raras na RBMMV, sendo que as duas primeiras apresentaram 0,10 sequiência individual de pegadas por $\mathrm{km}$ percorrido e as demais 0,07, 0,04 e 0,03, respectivamente (Quadro 2). Tamanduá-mirim mostrou-se mais raro que tamanduábandeira (Myrmecophaga tridactyla Linnaeus, 1758), fato que também foi observado no Parque Nacional das Emas (RODRIGUES et al., 2002).

\subsubsection{Espécies comuns}

Com base no índice de abundância obtido (Quadro 2), as seguintes espécies foram consideradas comuns na RBMMV: gambá (Didelphis albiventris), tamanduábandeira (M. tridactyla), tatu-peba (Euphractus sexcinctus (Linnaeus, 1758)), lobinho (Cerdocyon thous (Linnaeus, 1766)), anta (Tapirus terrestris (Linnaeus, 1758)), paca (Cuniculus paca (Linnaeus, 1766)), gato-do-matopequeno (Leopardus tigrinus) e jaguatirica (Leopardus pardalis (Linnaeus, 1758)); as duas primeiras apresentaram 0,50 seqüência individual de pegadas por $\mathrm{km}$ percorrido; e as demais, 0,31, 0,30, 0,24,0,15,0,13e0,12, respectivamente. Embora rara em muitas regiões do Cerrado, como no Parque Nacional das Emas (RODRIGUES et al., 2002), a jaguatirica mostrou-se comum na área de estudo. No entanto, por se apresentar como potencial predador de animais domésticos, como galinhas, não é descartada a possibilidade de que essa espécie seja abatida por moradores do entorno da RBMMV que criam aves soltas.

Quadro 2 - Abundância relativa (média de seqüências individuais de pegadas por km percorrido), desvio-padrão e status local das espécies de mamíferos registradas na Reserva Biológica Municipal "Mário Viana”, Nova Xavantina, MT

Table 2 -Relative abundance (average of individual's sequence tracks per km traveled), standard deviation and local status of the mammal species registered in the 'Mario Viana' Municipal Biological Reserve, Nova Xavantina, MT

\begin{tabular}{|c|c|c|c|}
\hline Espécie & Abundância relativa & Desvio padrão & Status local \\
\hline Didelphis albiventris & 0,50 & 0,54 & comum \\
\hline Myrmecophaga tridactyla & 0,50 & 0,23 & comum \\
\hline Tamandua tetradactyla & 0,10 & 0,20 & rara \\
\hline Dasypus novemcinctus & 1,02 & 0,76 & abundante \\
\hline Euphractus sexcinctus & 0,31 & 0,38 & comum \\
\hline Priodontes maximus & 0,03 & 0,10 & rara \\
\hline Cerdocyon thous & 0,30 & 0,25 & comum \\
\hline Pseudalopex vetulus & 0,03 & 0,10 & rara \\
\hline Procyon cancrivorus & 0,04 & 0,12 & rara \\
\hline Eira barbara & 0,03 & 0,10 & rara \\
\hline Herpailurus yaguarondi & 0,07 & 0,15 & rara \\
\hline Leopardus pardalis & 0,12 & 0,23 & comum \\
\hline Leopardus tigrinus & 0,13 & 0,18 & comum \\
\hline Puma concolor & 0,01 & 0,07 & rara \\
\hline Tapirus terrestris & 0,24 & 0,25 & comum \\
\hline Pecari tajacu & 0,84 & 1,04 & abundante \\
\hline Mazama americana & 0,10 & 0,20 & rara \\
\hline Mazama gouazoubira & 0,62 & 0,33 & abundante \\
\hline Hydrochaeris hydrochaeris & 0,01 & 0,07 & rara \\
\hline Cuniculus paca & 0,15 & 0,21 & comum \\
\hline Dasyprocta azarae & 2,26 & 0,75 & abundante \\
\hline Sylvilagus brasiliensis & 1,29 & 0,49 & abundante \\
\hline
\end{tabular}

R. Árvore, Viçosa-MG, v.30, n.4, p.669-678, 2006 
A presença de gato-do-mato-pequeno foi confirmada na RBMMV, por meio de captura. No entanto, seu índice de abundância pode estar superestimado, pois suas pegadas podem ser confundidas com as de gatodoméstico (Felis catus (Linnaeus, 1758)) (BECKER e DALPONTE, 1991), que já foi visto usando a área da reserva. Apesar de estar ameaçado de extinção (MMA, 2003), o tamanduá-bandeira mostrou-se comum na RBMMV, assim como no Parque Nacional das Emas (RODRIGUES et al., 2002). Esses dados devem estar refletindo o padrão de distribuição dessa espécie na região de Nova Xavantina, considerando-se que é comum encontrar indivíduos desse táxon em áreas de pastagens e também atropelados nas rodovias (DALPONTE, observação pessoal).

\subsubsection{Espécies abundantes}

De acordo com seus índices de abundância (Quadro 2), cutia (Dasyprocta azarae), tapeti (Sylvilagus brasiliensis), tatu-galinha (Dasypus novemcinctus), cateto (Pecari tajacu (Linnaeus, 1758)) e veadocatingueiro (Mazama gouazoubira (G. Fischer, 1814)) foram classificados como abundantes na RBMMV, apresentando 2,26, 1,29, 1,02, 0,84 e 0,62 seqüência(s) individual(is) de pegadas por $\mathrm{km}$ percorrido, respectivamente. Na RBMMV vive pelo menos um grupo de catetos, composto por cerca de seis indivíduos, sendo registrados principalmente nas áreas próximas ao córrego Bacaba. Cateto também se mostrou abundante durante um estudo realizado no leste da Amazônia (LOPES e FERRARI, 2000). Num estudo realizado em reservas de Floresta Atlântica no Espírito Santo, tapetí também apresentou elevada abundância (CHIARELLO, 1999). No entanto, não há registros dessa espécie no Parque Nacional das Emas, onde predominam "habitats" abertos de Cerrado. Veado-catingueiro, que é uma espécie generalista e bastante tolerante às alterações ambientais (DOTTA, 2005), foi considerado raro no Parque Nacional das Emas (RODRIGUES et al., 2002).

\subsection{Diversidade de espécies}

O índice de diversidade de Shannon-Wienner $\left(H^{\prime}\right)$ encontrado neste estudo, baseando-se nos dados de abundância registrados no transecto (Quadro 2), foi 2,40 , podendo ser considerado significativo, já que usualmente esse índice ocorre entre 1,5 e 3,5 (MAGURRAN, 1988). Durante um estudo realizado com mamíferos no leste da Amazônia brasileira, Lopes e Ferrari (2000) observaram que o $H^{\prime}$ diminuiu com aumentos nos distúrbios florestais e na pressão de caça, variando de 0,98 a 2,16, de acordo com o grau de perturbação do ambiente. Em outro estudo, realizado no Parque Nacional da Tijuca (3.300 ha), Rio de Janeiro (RJ), o $H^{\prime}$ de quirópteros foi 2,00 (ESBÉRARD, 2003). Num trecho do Alto Rio São Francisco, no Município de Três Marias (MG), o $H^{\prime}$ 'de peixes encontrado por Alvim (1999) foi 1,21. Numa avaliação da diversidade $\left(H^{\prime}\right)$ de Sphingidae (Lepidoptera) em áreas de brejo e caatinga no Estado da Paraíba foram encontrados índices de 2,32 e 1,65, respectivamente (GUSMÃO e CREÃO-DUARTE, 2004).

\section{CONCLUSÕES}

Apesar de pequena, a RBMMV desempenha importante papel para a conservação da mastofauna da região de Nova Xavantina, MT. Assim, funciona como área de refúgio, já que a maioria das áreas de entorno foi transformada em pastagem exótica, para a criação de gado.

As espécies de mamíferos presentes na RBMMV não estão totalmente protegidas, já que é comum encontrar cachorro (Canis familiaris (Linnaeus, 1758)) e gato (Felis catus) domésticos percorrendo e, até mesmo, perseguindo animais silvestres dentro da RBMMV. A presença desses animais domésticos representa uma potencial ameaça à mastofauna local, considerandose que eles são potenciais transmissores de doenças, têm a capacidade de predar animais silvestres e, além disso, são competidores diretos por recursos alimentares. A ação de caçadores e a ocorrência de incêndios incidentais, provenientes das pastagens adjacentes, também representam ameaça à sobrevivência dos mamíferos da RBMMV.

Recomendam-se novos estudos de longa duração com mamíferos na RBMMV, visando monitorar flutuações populacionais e taxas de extinção e, ou, incremento de espécies.

\section{AGRADECIMENTOS}

Aos nossos amigos Geane Brizzola dos Santos e Leo Soares, pela inestimável contribuição durante a realização deste estudo; Vanessa Cardoso Antunes, pelo auxílio na coleta de dados durante algumas idas à campo; Ronivon Mendes Duarte por ajudar na medição do transecto; e Prof. Elias Silva (Universidade Federal de Viçosa) pela leitura crítica do manuscrito.

R. Árvore, Viçosa-MG, v.30, n.4, p.669-678, 2006 


\section{REFERÊNCIAS BIBLIOGRÁFICAS}

AlviM, M. C. C. Composição e alimentação da ictiofauna em um trecho do Alto rio São Francisco, município de Três Marias - MG. 1999. 86 f. Dissertação (Mestrado em Ecologia e Recursos Naturais) - Universidade Federal de São Carlos, São Carlos, 1999.

BECKER, M.; DALPONTE, J. C. Rastros de mamíferos silvestres brasileiros: um guia de campo. Brasília: Universidade de Brasília, 1991. 180 p.

BONVICINO, C. R..; LINDBERGH, S. M.; MAROJA, L. S. Small non-flying mammals from conserved and altered areas of Atlantic Forest and Cerrado: comments on their potential use for monitoring environment. Brazilian Journal of Biology, v. 62, n. 4B, p. 765-774, 2002.

BROWER, J. E.; ZAR, J. H. Field \& laboratory methods for General Ecology. Dubuque: Wm. C. Brown Publishers, 1984. 226 p.

CHIARELLO, A. G. Effects of fragmentation of the Atlantic forest on mammal communities in southeastern Brazil. Biological Conservation, v. 89, p. 71-82, 1999.

CUELLAR, E.; NOSS, A. Conteo de huellas en brechas barridas: un índice de abundancia para mamíferos. Ecologia en Bolivia, v. 30, p. 55-67, 1997.

DALPONTE, J. C. Diet of the hoary fox, Lycalopex vetulus, in Mato Grosso, Central Brazil. Mammalia, v. 61, n. 4, p. 537-546, 1997.

DALPONTE, J. C. História natural, comportamento e conservação da raposa-do-campo, Pseudalopex vetulus (Canidae). 2003. 179 f. Tese (Doutorado em Biologia Animal) - Universidade de Brasília, Brasília, 2003.

DOTTA, G. Diversidade de mamíferos de médio e grande porte em relação à paisagem da bacia do rio Passa-cinco. 2005. 116 f. Dissertação (Mestrado em Ecologia de Agroecossistemas) - Escola Superior de Agricultura “Luiz de Queiroz”, Piracicaba, 2005.

R. Árvore, Viçosa-MG, v.30, n.4, p.669-678, 2006
ESBÉRARD, C. E. L. Diversidade de morcegos em área de Mata Atlântica regenerada no sudeste do Brasil. Revista Brasileira de

Zoociências, v. 5, n. 2, p. 189-204, 2003.

FELFILI, J. M.; SILVA JUNIOR, M. C.; NOGUEIRA, P. E. Levantamento da vegetação arbórea na região de Nova Xavantina, MT. Boletim do Herbário Ezechias Paulo Heringer, v. 3, p. 63-81, 1998.

FONSECA, G. A. B. et al. Lista anotada dos mamíferos do Brasil. Conservation Biology, v. 4, p. 1-38, 1996.

GUSMÃO, M. A. B.; CREÃO-DUARTE, A. J. Diversidade e análise faunística de Sphingidae (Lepidoptera) em área de brejo e caatinga no estado da Paraíba, Brasil. Revista Brasileira de Zoologia, v. 21, n. 3, p. 491-498, 2004.

HAYWARD, G. F.; PHILLIPSON, J. Community structure and functional role of small mammals in ecosystems. In: STODDART, D. M. (Ed.).

Ecology of small mammals. London: Chapman and Hall, 1979. p. 135-211.

IUCN - The World Conservation Union. Red list categories and criteria: version 3.1. Cambridge: IUCN - Species Survival Commission, 2001. 32 p.

LOPES, M. A.; FERRARI, S. F. Effects of Human Colonozation on the abundance and diversity of mammals in eastern Brazilian Amazônia. Conservation Biology, v. 14, n. 6, p. $1658-1665,2000$.

LUDWIG, J. A.; REYNOLDS, J. F. Statistical Ecology: a primer on methods and computing. New York: John Wiley \& Sons, 1988. 324 p.

MAGURRAN, A. E. Ecological diversity and its measurement. Oxford: Princeton University Press, 1988. 179 p.

MARIMON, S. M.; VARELLA, R. F.; MARIMON JÚNIOR, B. H. Fitossociologia de uma área de cerrado de encosta em Nova Xavantina, Mato Grosso. Boletim do Herbário Ezechias Paulo Heringer, v. 3, p. 82-101, 1998. 
MARINHO-FILHO, J.; RODRIGUES, F. H. G.; GUIMARÃES, M. M.; REIS, M. L. Os mamíferos da Estação Ecológica de Águas Emendadas, Planaltina, DF. In: MARINHO-FILHO, J.; RODRIGUES, F. H. G.; GUIMARÃES, M. M. (Eds). A fauna de vertebrados da Estação Ecológica de Águas Emendadas História Natural e Ecologia em um fragmento de Cerrado do Brasil Central. Brasília: SEMATEC/IEMA, 1998. p. 34-63.

\section{MINISTÉRIO DO MEIO AMBIENTE - MMA.}

Espécies da fauna brasileira ameaçadas de extinção. Instrução Normativa ${ }^{\circ}$ 3, de 27 de maio de 2003, publicada no Diário Oficial da União n 101 , de 28 de maio de 2003. Seção 1. p. 88-97.

NICHOLS, J. D.; CONROY, M. J. Techniques for estimating abundance and species richness. In: WILSON, D. E. et al. (Eds.). Measuring and monitoring biological diversity: standard methods for mammals. Washington: Smithsoniam Institution Press, 1996. p. 177-234.

NIMER, E. Climatologia do Brasil. Rio de Janeiro: IBGE, 1989. 422 p.

PARDINI, R. et al. Levantamento rápido de mamíferos terrestres de médio e grande porte. In: CULLEN JÚNIOR, L.; RUDRAN, R.;

VALLADARES-PADUA, C. (Orgs). Métodos de estudos em biologia da conservação e manejo da vida silvestre. Curitiba: Universidade Federal do Paraná, 2003. p. 181-201.
PINE, R. H.; BISHOP, I. R.; JACKSON, R. J. Preliminary list of mammals of the Xavantina/ Cachimbo expedition (Central Brazil).

Transactions of the Royal Society of Tropical Medicine and Hygiene, v. 64, n. 5, p. 668-670, 1970.

RADAMBRASIL. Levantamento dos Recursos Naturais. Ministério das Minas e Energia: 25, Folha SD - 22 Goiás, 1981.

RODRIGUES, F. H. G. et al. Composição e caracterização da fauna de mamíferos do Parque Nacional das Emas, Goiás, Brasil. Revista Brasileira de Zoologia, v. 19, n. 2, p. 589600, 2002

SCOSS, L. M. et al. Uso de parcelas de areia para o monitoramento de impacto sobre a riqueza de espécies de mamíferos. Revista Árvore, v. 28, p. 121-127, 2004.

STANDER, P. E. et al. Tracking and the interpretation of spoor: a scientifically sound method in ecology. Journal of Zoology, v. 242, p. 329-341, 1997.

WILKIE, D. S.; FINN, J. T. Slash-burn Cultivation and Mammal Abundance in the Ituri Forest, Zire. Biotropica, v. 22, n. 1, p. 90-99, 1990.

WWF. Manejo de Fauna na Reserva Xavante Rio das Mortes, MT. Brasília: 2000.64 p. 